\title{
A closer look into ECR 2020 on hybrid, molecular, and translational imaging
}

\author{
Thomas H. Helbich ${ }^{1}$ (D)
}

Received: 1 March 2020 / Accepted: 5 May 2020 / Published online: 18 May 2020

(C) European Society of Radiology 2020

Medical imaging has transformed disease diagnosis and rendered treatment of several medical conditions more effective. Increased awareness of the use of imaging devices due to advancements in technologies is driving the global market for medical imaging. The global market is expected to reach $\$ 40.56$ billion in 2021, which is a dramatic increase of more than $\$ 10$ billion since 2015 (https://www.itnonline.com/ article/global-medical-imaging-trends). In the field of medical imaging science, the concept of multiplexing and multimodality is providing the driving force for the development of next-generation imaging technologies. Hybrid imaging that incorporates two or more imaging modalities, imaging of tissue function, and imaging at the cellular and molecular levels translates rapidly from bench to bedside.

This article provides a brief overview of key research topics in the field of hybrid, molecular, and translational imaging, which were presented at the European Congress of Radiology (ECR) 2020 and highlights the challenges of medical imaging-based research.

Metabolic reprogramming plays a key role in the origin or progression of many diseases. Clinicians and patients can, therefore, benefit from a readily available, noninvasive, molecular imaging modality that provides detailed metabolic information. The most common, clinically used imaging strategy for interrogating metabolism is hybrid imaging with PET/ $\mathrm{CT}$ or PET/MRI. In particular, $18 \mathrm{~F}$ fluorodeoxyglucose (FDG) PET/CT has widespread applications and has emerged as an indispensable tool in the detection, staging, treatment monitoring, and identification of recurrent disease. At this year's ECR, several studies addressed the clinical utility of

Thomas H. Helbich

thomas.helbich@meduniwien.ac.at

1 Department of Biomedical Imaging and Image-guided Therapy, Division of Molecular and Gender Imaging, Medical University of Vienna \& General Hospital, Waehringer Guertel 18-20, Floor 7F, 1090 Vienna, Austria targeted radiotracers and showed that, in addition to $18 \mathrm{~F}$ FDG an array of novel tracers, such as $18 \mathrm{~F}$ fluoroethyltyrosine, $18 \mathrm{~F}$ choline, and $68 \mathrm{Ga}$-PSMA, allow more precise insight into different diseases, may serve as independent prognostic imaging biomarkers, and can help to individualize clinical management and decision-making. These techniques span the spectrum from oncologic imaging, to imaging of inflammation and neurodegenerative diseases. Considering the enormous clinical impact, a next generation of PET/CT scanner has entered the field.

The work by Abdelhafez et al. aimed to characterize different aspects of this scanner's impact on the visualization of small structures through a semiquantitative evaluation [1]. They showed that biodistribution values in small structures are higher than those usually seen from standard scanners. In addition, this next generation of PET/CT scanner offers total body scanning, enables ultra-low patient doses, and produces high image resolution in a very short time. It is, therefore, expected that this next-generation technology will soon become the standard of care.

In addition to the role of PET/MRI/CT, exciting new magnetic resonance spectroscopy (MRS) strategies have been presented. 13C-pyruvate MRS is an emerging molecular imaging method that allows rapid, noninvasive, and pathway-specific investigation of dynamic metabolic and physiologic processes that were previously inaccessible to imaging. Ursprung et al. assessed the role of hyperpolarized 13C-pyruvate MRI in renal tumors and demonstrated that 13C-pyruvate MRI allows insight into metabolic intratumoral heterogeneity and that imaging-based lactate levels correlate with steady-state tissue lactate concentrations [2]. 13C-pyruvate MRI may guide biopsies to regions of metabolically aggressive areas of the tumor.

Another novel approach that meets the current needs for metabolic imaging was presented by Hesse et al. [3]. Like 13C, deuterium-labeled substrates can be used to study metabolism with MRS in vivo. They applied a 3D deuterium MRI pulse sequence with a time resolution of 5 minutes, 
following a bolus injection of [2,3-2H2] fumarate to detect early evidence of cell death in three different tumor models before and $48 \mathrm{~h}$ or $24 \mathrm{~h}$ after treatment with a chemotherapeutic or a novel targeted agent, respectively. These authors showed that deuterium MRS can provide quantitative and spatially resolved measurements of cell death imaging, thus highlighting the clinical potential for assessment of early treatment response. Data indicate that hyperpolarized 13Cpyruvate MRI and deuterium MRS are poised to become widespread methods for metabolic imaging in both research and in the clinic.

Another key research topic is nanomedicine, with its promising potential to improve anticancer therapy in particular. Nanoparticles can work as carriers of drugs, nucleic acids, and imaging agents, or, they can sensitize cells to radiation and are currently at various stages of investigation, translation, and clinical implementation. Yao et al. reported on the development of a peptide-targeted gold nanorod, called PepSq@AuNRs, which is sensitive to fluorescent, photoacoustic, and computed tomography (FL/PA/CT) imaging and allows trimodal, non-invasive, accurate detection and riskstratification of breast cancer [4]. Pep- Sq@ AuNRs specifically binds to extradomain-B fibronectin, a marker for epithelialtomesenchymal transition, which is a biological process associated with tumor invasion, metastasis, and drug resistance, with a new theranostic approach presented by Wang et al. [5]. In light of upregulated cathepsin-B in breast cancer, they synthesized a targeted nearinfrared activatable probe called PepSQ@USPIO for MRI/fluorescent/photoacoustic (MRI/NIR FL/PA) imaging-guided photodynamic therapy. In vitro and in vivo studies showed that this tumor-specific theranostic probe can precisely diagnose and exert further selective treatment.

Helfen et al. focused on fluorescence reflectance imaging of the protein S100A8/A9, which is released by activated tumor-infiltrating monocytes and forms a characteristic inflammatory tumor microenvironment associated with a poor prognosis [6]. These investigators demonstrated that S100A9specific in vivo imaging reflects tumor malignancy and may serve as a biomarker for tumor microenvironment formation and activity. It can be expected that the use of nanoparticles in the field of medical imaging willrevolutionize the way we detect and treat disease in the future.

Considering the above-described key research topics, a clear trend is evident toward effective implementation of hybrid imaging (PET/CT, PET/MRI), the development of imaging biomarkers, targeted tracers, and smart nanoparticles, as well as real-life visualization of metabolic processes. Nevertheless, we have to acknowledge that we are just entering a new era of personalized and precision medicine, with the initial results presented at ECR 2020. We should recognize that only the tip of the iceberg with regard to molecular and translational preclinical imaging was presented at the ECR
2020. Although visionary and revolutionary concepts in the field of preclinical medical imaging sciences were presented at other congresses by non-radiologists, spanning the spectrum from biology, chemistry, physics, bioengineering, computer science, and many more, it should be noted that the European Society of Radiology (ESR) or its sub-societies, such as ESHIMT, make a conscious effort to foster specific educational and training programs that evolve and disseminate knowledge about pre-clinical imaging sciences in Europe and across the world. Moreover, EIBIR, a non-profit limited liability company that was founded by the ESR, aims to coordinate and support the development of medical imaging and the dissemination of knowledge with the goal of improving the diagnosis, treatment, and prevention of disease.

Last, but not least, the ESR journal family, which includes European Radiology, European Radiology Experimental and Insight into Imaging offers a profound base from which to publish exciting research articles. We, therefore, must ask ourselves why - despite these efforts - only a minority of young radiologists are involved in this breathtaking preclinical and translational field of research that includes hybrid, molecular, and translational imaging. We as senior researchers and mentors have the responsibility to open new avenues, provide opportunities, and guide future research projects. This young generation of radiologists is our future. Let's give them time to fulfill their research dreams!

Funding information This work has received no funding.

\section{Compliance with ethical standards}

Guarantor Not applicable.

Conflict of interest The author declares no conflict of interest.

Statistics and biometry No complex statistical methods were necessary for this paper.

Informed consent Not applicable

Ethical approval Institutional Review Board approval was not required because the paper is an Editorial.

Study subjects or cohorts overlap Not applicable

Methodology

-Not applicable as this work is an editorial.

\section{References}

1. Abdelhafez Y, Omidvari N, Spencer B, Badawi RDB, Cherry S, Nardo L (2020) Initial evaluation of 18F-FDG biodistribution in healthy and oncology subjects scanned using the UEXPLORER total-body PET/CT/. ECR 2020 Book of Abstracts. Insights Imaging. https://doi.org/10.1186/s13244-020-00851-0 
2. Ursprung S, Woitek RA, McLean MA et al (2020) Virtual metabolic biopsies using hyperpolarised carbon-13 MRI to unravel metabolic heterogeneity in renal tumours. ECR 2020 Book of Abstracts. Insights Imaging. https://doi.org/10.1186/s13244-020-00851-0

3. Hesse F, Somai V, Kreis F, Bulat F, Brindle K (2020) Assessing tumour cell death in vivo using deuterium magnetic resonance spectroscopic imaging. ECR 2020 Book of Abstracts. Insights Imaging. https://doi.org/10.1186/s13244-020-00851-0

4. Yao D, Wang Y, Wang D (2020) Extradomain-B fibronectintargeting nanoprobe for lara-floresFL/PA/CT trimodal accurate imaging and risk-stratification of breast cancer. ECR 2020 Book of abstracts. Insights Imaging. https://doi.org/10.1186/s13244-02000851-0
5. Wang Y, Yao D, Wang D (2020) Cathepsin B-activated nanoparticles for multimodal imaging-guided photodynamic therapy to breast cancer. ECR 2020 Book of abstracts. Insights Imaging. https://doi. org/10.1186/s13244-020-00851-0

6. Helfen A, Rieß J, Schnepel A et al (2020) Target-specific in vivo imaging of tumourimmune interaction: evaluation of the regulatory protein S100A9. ECR 2020 Book of Abstracts. Insights Imaging https://doi.org/10.1186/s13244-020-00851-0

Publisher's note Springer Nature remains neutral with regard to jurisdictional claims in published maps and institutional affiliations. 